\title{
sciendo
}

\section{Different Shoulder Exercises Affect the Activation of Deltoid Portions in Resistance-Trained Individuals}

\author{
by \\ Yuri A. C. Campos ${ }^{1,2}$, Jeferson M. Vianna², Miller P. Guimarães ${ }^{1,3}$, \\ Jorge L. D. Oliveira², Claudio Hernández-Mosqueira ${ }^{4,5}$, Sandro F. da Silva ${ }^{1}$, \\ Paulo H. Marchetti ${ }^{6}$
}

The aims of this study were to compare muscle activity of the anterior deltoid, medial deltoid, and posterior deltoid in the bench press, dumbbell fly, shoulder press, and lateral raise exercises. Thirteen men experienced in strength training volunteered for the study. Muscle activation was recorded during maximum isometric voluntary contraction (MVIC) for data normalization, and during one set of 12 repetitions with the load of $60 \% 1 R M$ in all exercises proposed. One-way repeated-measures ANOVA with Bonferroni's posthoc was applied using a 5\% significance level. For anterior deltoid, the shoulder press (33.3\% MVIC) presented a significantly higher level of activation when compared to other exercises. Also, no significant difference was found between the bench press $(21.4 \%$ MVIC), lateral raise (21.2\% MVIC), and dumbbell fly (18.8\% MVIC). For the medial deltoid, the lateral raise (30.3\% MVIC) and shoulder press (27.9\% MVIC) presented a significantly higher level of activity than the bench press (5\% MVIC) and dumbbell fly (3.4\% MVIC). Besides, no significant difference was found between the bench press and the dumbbell fly. For the posterior deltoid, the lateral raise (24\% MVIC) presented a significantly higher level of activation when compared to other exercises. For the posterior deltoid portion, the shoulder press (11.4\% MVIC) was significantly more active than the bench press (3.5\% MVIC) and dumbbell fly (2.5\% MVIC). Moreover, no significant difference was found between the bench press and the dumbbell fly. In conclusion, the shoulder press and lateral raise exercises showed a higher level of muscle activation in the anterior deltoid and medial deltoid when compared to the bench press and dumbbell fly exercises.

Key words: EMG, strength training, glenohumeral joint, deltoid muscle, upper body.

\section{Introduction}

The deltoid is considered a primary motor muscle in many upper body strength training (ST) exercises (Andersen et al., 2014; Botton et al., 2013; Franke et al., 2015; Welsch et al., 2005; Wilk et al., 2019). Due to its triangular shape, the deltoid muscle is commonly subdivided into anterior, medial and posterior portions (Botton et al., 2013) being responsible for several movements of the glenohumeral joint such as shoulder abduction (medial deltoid, anterior and posterior) (Hall, 2006; Houglum and Bertoti, 2011), shoulder flexion and horizontal adduction

1 - Studies Research Group in Neuromuscular Responses, University of Lavras, Lavras, Brazil.

2 - Postgraduate Program of the Faculty of Physical Education and Sports of the University of Juiz de fora, University of Juiz de Fora, Juiz de Fora, Brazil.

3 - Postgraduate in Human Movement and Rehabilitation Sciences, University of São Paulo, Santos, Brazil.

4 - Department of Physical Activity Sciences, University of Los Lagos, Puerto Montt, Chile.

5 - AFSYE research group, Adventist University of Chile, Chile.

6 - Department of Kinesiology, California State University, Northridge, CA, USA. 
(anterior deltoid) (Hall, 2006; Houglum and Bertoti, 2011), as well as shoulder extension and horizontal abduction (posterior deltoid) (Hall, 2006; Houglum and Bertoti, 2011).

The use of different exercises with different mechanical strains has been highly recommended for the complete development of different deltoid portions. In this sense, priority exercises such as the bench press (McCaw and Friday, 1994; Schick et al., 2010; Welsch et al., 2005; Wilk et al., 2019), lat pulldown (Andersen et al., 2014; Vilaça-Alves et al., 2014), seated row (Botton et al., 2013; Franke et al., 2015), and complementary exercises such as the lateral raise (Botton et al., 2013), shoulder press (Saeterbakken and Fimland, 2013), and reverse pec deck fly (Botton et al., 2013; Franke et al., 2015) can be used. However, the importance of including complementary exercises in ST routines, as well as how these different exercises affect the muscle recruitment of the deltoid portions remain still unclear.

Some studies using surface electromyography (sEMG) have been proposed to verify differences in muscle activity during priority and complementary exercises for the upper (Botton et al., 2013; Franke et al., 2015) and lower (Ema et al., 2016; Wright et al., 1999) limbs. Wright et al. (1999) found greater muscle activation in the semitendinosus and biceps femoris muscles during knee flexion when compared to squats. Ema et al. (2016) found greater muscle activity in the rectus femoris during knee extension compared to the leg press. As for the medial portion of the deltoid, Botton et al. (2013) reported higher muscle activity during the lateral raise exercise with a cable/dumbbell than the shoulder press. Regarding the posterior portion of the deltoid, Botton et al. (2013) and Franke et al. (2015) reported higher muscle activation of this muscle portion by the reverse pec deck fly exercise than a seated row or inclined pulldown. Additionally, for the anterior portion of the deltoid, Botton et al. (2013) did not observe differences in muscle activation between the smith machine shoulder press and the bench press.

Therefore, considering that exercise selection is one of the important variables to maximize acute responses and chronic adaptations (Grgic et al., 2018) and that muscle involvement plays a key role in your choice (Stastny et al., 2017), the objective of this study was to compare the muscle activity of anterior, medial, and posterior portions of the deltoid in the bench press, dumbbell fly, shoulder press, and lateral raise. A thorough understanding of how each portion of the deltoid is involved in each exercise might assist coaches and physiotherapists in choosing and using the most appropriate exercise in ST and rehabilitation programs.

\section{Methods}

\section{Experimental Approach to the Problem}

To investigate muscle activity of the anterior deltoid (AD), medial deltoid (MD), and posterior deltoid (PD) during the bench press (BP), dumbbell fly (DF), shoulder press (SP), and lateral raise (LR), five sessions were conducted separated by a 48-hour interval. During the first session, participants were evaluated for body mass, height, and body fat and were informed about all procedures that would involve data collection. During the second and third sessions, participants performed the test and retest of onerepetition maximum (1RM) in the proposed exercises. During the fourth session, data of muscle activation during the proposed exercises were collected. After a 15-min rest interval, participants performed a maximum voluntary isometric contraction (MVIC) in each exercise for subsequent normalization of the EMG signal. The evaluated exercises were performed randomly in the second, third, and fourth sessions.

\section{Participants}

This study included 13 healthy male volunteers (age $24.50 \pm 4.46$ years, fat content $13.80 \pm 4.92 \%$, body height $1.76 \pm 0.06 \mathrm{~m}$, body mass $78.40 \pm 15.30 \mathrm{~kg}$, $1 \mathrm{RM}$ testing $-\mathrm{BP} 111.50 \pm$ $7.12 \mathrm{kgf}$, DF $70.38 \pm 5.18 \mathrm{kgf}$, SP $55.53 \pm 4.63 \mathrm{kgf}$, and LR $34.90 \pm 2.98 \mathrm{kgf}$ ) with $3.58 \pm 2.90$ years of ST experience, who performed the proposed exercises regularly in their training programs. The exclusion criteria were the presence of any type of bone, joint or muscle injuries that compromised the total or partial performance of the movements, or participants who were not familiar with the proposed exercises. All procedures were approved by the local ethics committee (protocol: \#0068/2010) following the Declaration of Helsinki. All participants signed a consent form for their participation in the study. 


\section{Evaluation}

Anthropometric evaluation

A stadiometer $\left(110 \mathrm{FF}\right.$, Welmy ${ }^{\circledR}$, Santa Bárbara d'Oeste, Brazil) was used for anthropometric evaluation and a four-pole bioimpedance device (Quantum BIA-II, RJL Systems ${ }^{\circledR}$, Clinton Township, USA) was used to estimate body fat composition.

One-repetition maximum (1RM) testing

The 1RM protocol followed recommendations proposed by the National Strength and Conditioning Association (Baechle and Earle, 2008). Some precautions were followed to reduce the possibility of errors in the determination of $1 \mathrm{RM}$ for each exercise: 1) participants were instructed to execute $\mathrm{BP}, \mathrm{DF}, \mathrm{SP}$, and LR exercises according to the National Strength and Conditioning Association manual (NCSA, 2008); 2) the correct technique was monitored during the execution of all exercises; 3 ) researchers gave feedback to participants on each exercise technique; 4) researchers encouraged participants during all exercises; and 5) exercises were interrupted if there was any modification in the execution technique. Participants warmed up with mild cardiovascular exercises for approximately five to 10 minutes. Subsequently, from the load reported by participants to perform sets of 8 to 12 maximum repetitions, the warm-up loads were calculated by an equation (Brzycki, 1993). Then, all participants executed a set of five repetitions of each exercise at 50\% 1RM, followed by two to three repetitions with a load corresponding to 60 and $80 \% 1 \mathrm{RM}$ as a specific warm-up. Participants executed a set of single repetitions with increasing weight to determine 1RM, and a 5-min rest interval between attempts. The 1RM was tested using maximally five attempts. These procedures were followed for all exercises proposed (Schoenfeld et al., 2016). A 30min recovery interval was used between exercises.

Measurements

Participants had their skin shaved, wiped, and cleaned using a cotton ball and isopropyl alcohol. Then, disk-shaped self-adhesive sEMG surface electrodes (2223 BR, 3M®, Campinas, Brazil) with a one-centimeter diameter $\mathrm{AgCl}$ capture surface were placed using the conductive gel in the presumable underlying muscle fiber direction at a center-to-center distance of approximately $2 \mathrm{~cm}$. Surface electrodes were placed on the evaluated muscles according to the SENIAM recommendations (Hermens et al., 1999) on the dominant side of each participant (Behm et al., 2005). For AD, electrodes were fixed at one finger width distal and anterior to the acromion in the direction of the line between the acromion and the thumb. For MD, the electrodes were fixed from the acromion to the lateral epicondyle of the elbow in the direction of the line between the acromion and the hand. For PD, the electrodes were centered in the area about two finger breaths behind the angle of the acromion in the direction of the line between the acromion and the little finger. Reference electrodes were properly placed on the bony process of the elbow. After electrodes fixation, participants' skin was marked with a special pen to avoid mistakes in electrode positioning between the fourth and fifth sessions. Electromyography (Miotool 400, Miotec ${ }^{\circledR}$, Porto Alegre, Brazil) with four input channels, 14 bits resolution, and the acquisition rate per channel of 2000 samples/s and an SDS-500 sensor with a maximum gain of 1000 times were used to collect electromyographic signals. The common-mode rejection ratio was $106 \mathrm{~dB}$ and impedance between each electrode pair was $<10^{12} \Omega$. All electromyograph channels were properly calibrated prior to data collection. The concentric and eccentric phases were evaluated during a set of 12 repetitions. Then the first two and last two repetitions were excluded on the signal (RAW) to avoid mechanical failure or neuromuscular fatigue. The remaining eight repetitions of the sEMG signal were filtered with a fourth-order Butterworth bandpass filter with zero phase delay and cut-off frequency between 20 and $500 \mathrm{~Hz}$, and the amplitude was calculated using the root mean square (RMS) with a 100 ms moving window. Specialized Miograph 2.0 Alpha 9 Build 5 software (Miotec ${ }^{\circledR}$ Equipamentos Biomédicos Ltda, Porto Alegre, Brazil) was used for data analysis and processing. The sEMG RMS was normalized by the MVIC peak previously obtained for each muscle portion of the deltoid muscle. Finally, normalized sEMG RMS signal (RMSn) means were used for subsequent analysis.

\section{Procedures}

Participants were instructed to abstain from strenuous exercise and to avoid alcohol and caffeine for 48 hours before the evaluations. Soon 
after the anthropometric assessments, at the beginning of the experimental session, all participants performed 20 repetitions of each exercise as a warm-up, with loads adjusted to $30 \%$ 1RM. After a three-minute rest interval, they executed one set of 12 repetitions of each proposed exercise with loads adjusted to $60 \%$ 1RM (Muyor et al., 2019) to collect muscle activity (sEMG) data. During all exercises, movement speed (cadence) was maintained at $(2 / 0 / 1 / 0)$, i.e. a 2-s eccentric phase, 0 -s i.e., no break in the transition phase, a 1-s concentric phase, and 0-s i.e., no rest before the next repetition (Schoenfeld et al., 2015; Wilk et al., 2018) using a digital metronome (DM90, Seiko®, Tokyo, Japan). Exercises were performed as follows: for the BP and DF exercises, participants remained supine in the specific bank. During the SP and LR, participants remained seated, with their backs resting on a specific bench set at $90^{\circ}$, and their feet flat on the floor. In the initial BP and DF positioning, the shoulders were horizontally abducted according to each participant's footprint, using $165 \%$ and $100 \%$ of the bi-acromial distance, respectively, with the elbows extended. In both, participants were instructed to lower the barbell and dumbbells until the shoulders reached a horizontal abduction of approximately $45^{\circ}$ below the trunk line (Van den Tillaar and Ettema, 2009). For the SP exercise, participants used a barbell and started with the shoulders abducted at $180^{\circ}$ (from the anatomical position) and the elbows extended. Thus, they were instructed to perform shoulder adduction along with an elbow flexion in eccentric action until approximately $135^{\circ}$ shoulder adduction was reached. Finally, for the LR exercise, participants were instructed to perform $90^{\circ}$ of shoulder abduction from the anatomical position. A 15-min recovery interval was used between exercises. For the exercises, the following materials and equipment were used: (a) a 1.30-m and $10 \mathrm{~kg}$ barbell was used for the BP, (b) two $40-\mathrm{cm}$ and $2 \mathrm{~kg}$ dumbbells were used for the DF and LR, (c) a 1.10-m and $8 \mathrm{~kg}$ barbell was used for the SP. An adjustable bench and bumper plates were used for all exercises. All equipment used was produced by Physicus ${ }^{\circledR}$ (Auriflama, Brazil).

Maximum voluntary isometric contraction (MVIC)

Fifteen minutes after the main session, participants performed MVIC in all proposed exercises (Golas et al., 2017a, 2017b). For the MVIC in the bench press, dumbbell fly, shoulder press, and lateral raise, a barbell and/or apparatus for performing the lateral raise and fly on the pulley, a specific bank with adjustable inclination, and chains that used to secure the barbell to the ground at the specific angles of each exercise, were used. To measure the MVIC, the participant held the barbell or apparatus in the following positions: (a) BP - the barbell grip width was $165 \%$ of the biacromial distance with $90^{\circ}$ horizontal shoulder abduction and approximately $90^{\circ}$ elbow flexion; (b) DF - $90^{\circ}$ horizontal abduction and approximately $90^{\circ}$ elbow flexion (the reference position for both abovementioned exercises was complete horizontal shoulder adduction with the trunk in dorsal decubitus); (c) SP - $90^{\circ}$ shoulder abduction (in conjunction with external shoulder rotation) and $110^{\circ}$ elbow flexion; and (d) LR - $90^{\circ}$ shoulder abduction. All positions were defined using a goniometer (Konex®, São Paulo, Brazil). Participants performed three sets of 5-s contractions with a recovery time of $30 \mathrm{~s}$ between sets and exercises. The value attributed to normalization was the highest value found during the MVIC for each of the muscles analyzed among the four exercises evaluated (Castelein et al., 2016).

Statistical Analysis

Shapiro-Wilk and Levene's tests were used for analyses of variance normality and homogeneity, and data were reported using mean and standard deviation. If the assumptions of normality and homogeneity of variance were fulfilled, a one-way ANOVA with repeated measures and a Bonferroni's posthoc were used to compare $\mathrm{AD}, \mathrm{MD}$, and $\mathrm{PD}$ muscle activity (RMSn) among BP, DF, SP, and LR exercises. The effect size was calculated according to the Cohen's test (d) using the following formula: $d=$ (group 1 mean - group 2 mean)/standard deviation. Effect size (d) was evaluated using the following criteria: $<0.35$ trivial; $0.35-0.80$ small; $0.80-1.50$ moderate; and $>1.5$ large, according to the classification of recreationally trained individuals proposed by Rhea (2004). To verify reproducibility for the 1RM test-retest the intraclass correlation coefficient (ICC) was applied. A significance level $(\alpha)$ of $5 \%$ was used as statistical evidence and SPSS software (20.0, IBM, Armonk, USA) was used for statistical analysis. 


\section{Results}

ICCs for the 1RM test-retest were between 0.930 and 0.950 . For the anterior deltoid muscle activity (RMSn) significant differences were observed between the SP and DF ( $p=0.001, d=$ 1.41 [moderate], $\Delta \%=14.5)$; the $\mathrm{SP}$ and $\mathrm{BP}(p=$ $0.002, d=1.14$ [moderate], $\Delta \%=11.9$ ); and the SP and LR $(p=0.003, d=1.25$ [moderate], $\Delta \%=12.1)$. However, no significant difference was found between the BP and DF ( $p=0.091, d=0.31$ [trivial], $\Delta \%=2.6)$; the BP and LR $(p=0.738, d=0.03$ [trivial], $\Delta \%=0.2)$; and the LR and DF $(p=0.698, d$ $=0.33$ [trivial], $\Delta \%=2.4)($ Figure 1$)$.

For the medial deltoid muscle activation (RMSn) significant differences were observed between the LR and DF ( $p=0.001, d=3.48$ [large], $\Delta \%=26.9) ;$ the LR and BP $(p=0.001, d=3.23$ [large], $\Delta \%=25.3) ;$ the SP and DF ( $p=0.001, d=$ 2.90 [large], $\Delta \%=24.5)$; and the SP and DF ( $p=$
$0.001, d=2.68$ [large], $\Delta \%=22.9$ ). However, no significant difference was found between the LR and SP ( $p=0.596, d=0.22$ [trivial], $\Delta \%=1.6)$ and the BP and DF ( $p=0.484, d=0.79$ [small], $\Delta \%=$ 2.4) (Figure 2).

For the posterior deltoid muscle activity (RMSn) significant differences were observed between the LR and DF ( $p=0.001, d=3.95$ [large], $\Delta \%=21.5)$; the LR and BP $(p=0.001, d=3.67$ [large], $\Delta \%=20.5)$; the LR and SP $(p=0.014, d=$ 2.12 [large], $\Delta \%=12.6) ;$ the SP and DF $(p=0.001, d$ $=3.43$ [large], $\Delta \%=8.9) ;$ and the SP and BP $(p=$ $0.001, d=2.73$ [large], $\Delta \%=7.9)$. However, no significant difference was found between the BP and DF $(p=0.667, d=0.59$ [small], $\Delta \%=1.0)$ (Figure 3).

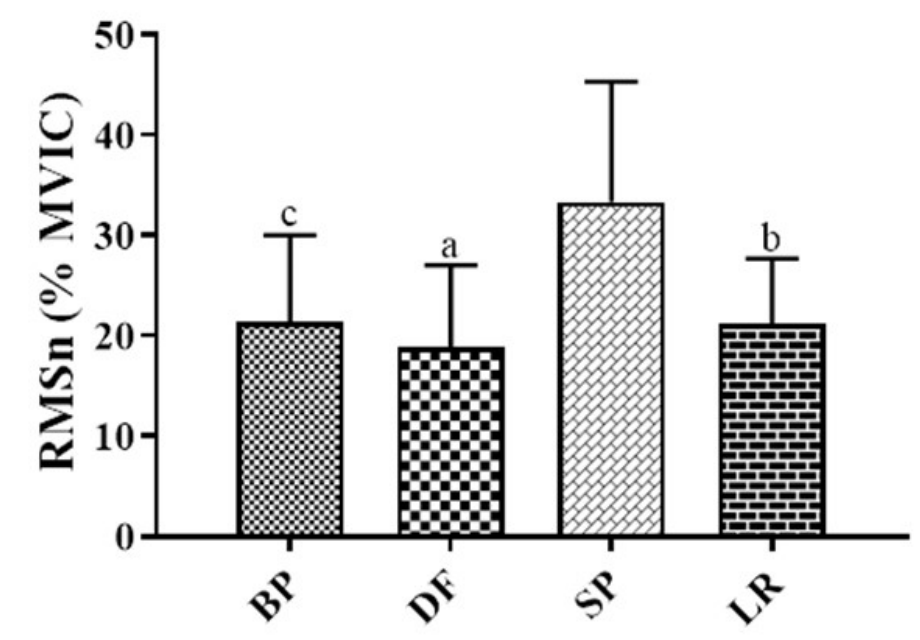

Figure 1. Mean and standard deviation of the anterior deltoid muscle activity (RMSn) for each exercise: the bench press $(B P)$, dumbbell fly $(D F)$, shoulder press $(S P)$, and lateral raise $(L R)$ exercises.

$a, b, c p \leq 0.05$, significant difference between the SP and DF, SP and BP and, SP and LR. 


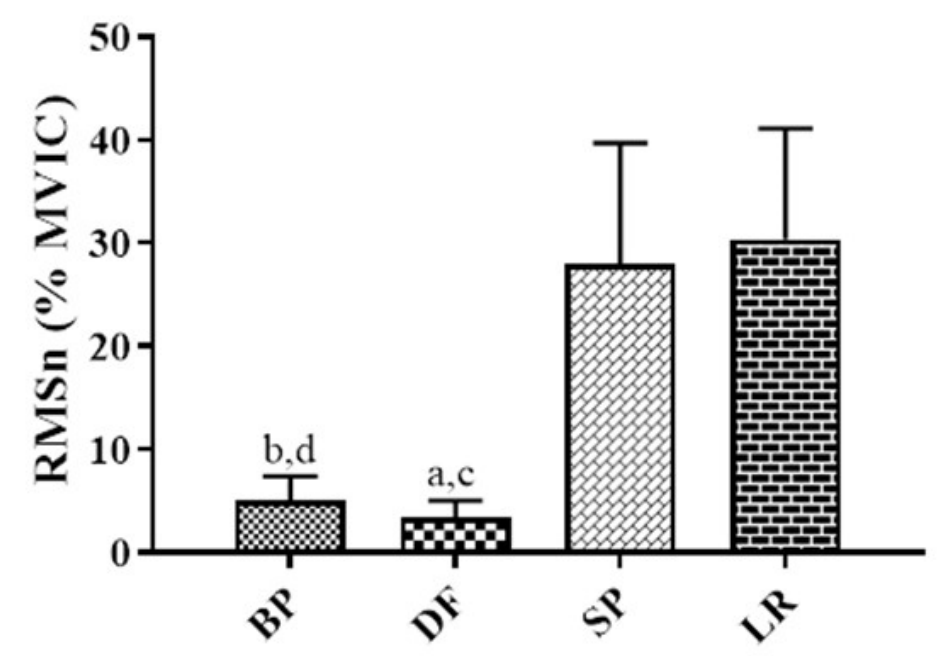

Figure 2. Mean and standard deviation of the medial deltoid muscle activity (RMSn) for each exercise: bench press $(B P)$, dumbbell fly $(D F)$, shoulder press $(S P)$, and lateral raise $(L R)$ exercises.

$a, b, c, d p \leq 0.05$, significant difference between the $L R$ and $D F, L R$ and BP,SP and DF, and $S P$ and $B P$.

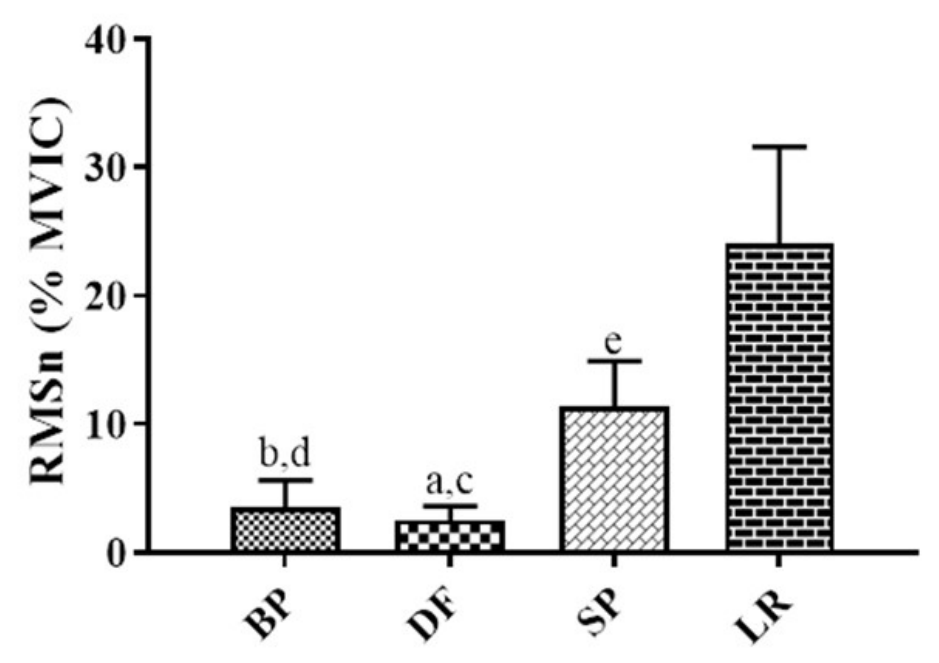

Figure 3. Mean and standard deviation of the posterior deltoid muscle activity (RMSn) for each exercise: the bench press $(B P)$, dumbbell fly $(D F)$, shoulder press $(S P)$, and lateral raise (LR) exercises.

$a, b, c, d, e p \leq 0.05$, significant difference between the $L R$ and $D F, L R$ and $B P, L R$ and SP, SP and DF, and $S P$ and $B P$. 


\section{Discussion}

The objective of this study was to compare the muscle activity of different deltoid portions (anterior [AD], medial [MD], and posterior $[\mathrm{PD}]$ for the $\mathrm{BP}, \mathrm{DF}, \mathrm{SP}$, and $\mathrm{LR}$ exercises. The main finding of the present study indicated that the shoulder press (SP) and lateral raise (LR) exercises presented a higher muscle activity for $\mathrm{AD}, \mathrm{MD}$, and $\mathrm{PD}$ when compared to the bench press (BP) and dumbbell fly (DF) exercises.

Exercises such as the BP and DF are frequently included in ST routines due to their large AD activation (Muyor et al., 2019; Welsch et al., 2005). Welsh et al. (2005) and Júnior et al. (2007) compared AD muscle activity response in the BP and DF exercises; however, they found no difference between them. Due to the magnitude and similarity of the $\mathrm{AD}$ muscle response when compared to the pectoralis major muscle, Júnior et al. (2007) suggested that specific exercises such as the SP are not necessary for the AD. In contrast, for the $\mathrm{AD}$ the present study showed higher muscle activation in the SP $(33.3 \%$ MVIC), BP (21.4\% MVIC), LR (21.2\% MVIC), and DF (18.8\% MVIC). In a similar study, Botton et al. (2013) found no significant difference in AD muscle activation between the Smith machine shoulder press, bench press, and peck deck exercises using 10RM workloads. However, the present study showed a significant difference between the SP and BP, and between the SP and DF (Figure 1), which might be partially explained by the use of free weights instead of a machine. Similarly, sEMG-based studies have shown greater muscle activation in free weight exercises when compared to machines in the AD muscle (McCaw and Friday, 1994; Schick et al., 2010; Schwanbeck et al., 2009). Thus, the SP can also be an effective exercise for the activation of the AD based on the movement of the shoulder (abduction associated with external rotation of the glenohumeral joint) (Liu et al., 1997), favoring the AD and MD activation, as shown in Figures 1 and 3.

As for the MD, the present study showed higher muscle activation in the LR (30.3\% MVIC), SP (27.9\% MVIC), BP (5\% MVIC), and DF (3.4\% MVIC). Based on the results of the present study, no significant difference between LR and SP exercises was observed. However, there was a significant difference between these exercises when compared to the BP and DF (Figure 2). Although this portion of the deltoid is considered the main glenohumeral abductor (Don Lehmkuhl and Smith, 1983; Hall, 1999) a similar muscle recruitment between the SP and LR may be related to high indices of muscular synergism between 50 and 90 degrees (Liu et al., 1997) reaching its maximum peak at 100 degrees of glenohumeral abduction (Wickham et al., 2010). Contrary to the results of the present study, Botton et al. (2013) reported higher MD muscle activity during cable/free weight LR compared to SP executed on a Smith machine. This difference may be related to the type of equipment used (free weight vs. machine) where greater activation is observed in primary motor and stabilizer muscles (McCaw and Friday, 1994; Schwanbeck et al., 2009). Thus, the use of the Smith machine in the SP (Botton et al., 2013) may have affected muscle activation by promoting greater stability during exercise execution.

PD muscle showed greater activation in the LR (24\% MVIC), SP (11.4\% MVIC), BP (3.5\% MVIC), and DF (2.5\% MVIC). The results of our study showed a significant difference for the LR compared to the BP, DF, and SP. Additionally, a significant difference was found for the SP compared to the BP, and DF (Figure 3). The increased PD muscle recruitment during the LR observed in the present study might be explained by its secondary and stabilizing function during glenohumeral joint abduction (Botton et al., 2013). Some studies suggested the use of more specific exercises, such as reverse the pec deck fly for a greater PD participation (Botton et al., 2013; Franke et al., 2015), but this exercise was not analyzed in the present study. In this sense, Botton et al. (2013) reported higher PD muscle activity during the reverse pec deck exercise compared to the LR.

A limitation of the study is the difficulty in comparing the workload (60\% 1RM) used in the present investigation to collect the sEMG signal with workloads used in other studies (Botton et al., 2013; Franke et al., 2015; McCaw and Friday, 1994; Schick et al., 2010; Schwanbeck et al., 2009). Although classic (McCaw and Friday, 1994) and current (Muyor et al., 2019) studies have used $60 \% 1 \mathrm{RM}$ workloads for sEMG signal collection, these results should be interpreted with caution as exercise intensity may modify the 
pattern of muscle recruitment (McCaw and Friday, 1994; Schick et al., 2010; Stastny et al., 2017). Besides, limitations regarding the use of sEMG signals during dynamic contractions (Stastny et al., 2017) and their comparisons between different exercises (Vigotsky et al., 2018) should be considered. Moreover, not evaluating the percentage of body fat in the specific regions where the surface electrodes were placed, which could affect electromyographic signal levels due to its low-pass filter characteristic, is another limitation. To reduce this problem, electromyographic signals were normalized by positioning electrodes on the same collection sites. In addition, there is a possibility that the data normalization process adopted in our study did not allow reaching the maximum contraction value for all studied muscles (Golas et al., 2018).

Complementary exercises for the anterior portion of the deltoid, such as the shoulder press, should be included in the ST routines to offer greater variability to the training program and to present greater muscle activation when compared to priority exercises such as the bench press and dumbbell fly. However, the inclusion of many sets of complementary exercises for this muscle portion should be viewed with caution, as it is often requested during priority exercises such as the bench press and dumbbell fly. Also, complementary exercises such as the lateral raise and shoulder press should be included in ST routines, because they have greater muscle activation in the medial portion of the deltoid when compared to priority exercises such as the bench press and dumbbell fly. Additionally, the inclusion of these exercises in the ST programs would be important to maintain muscle activation of the medial deltoid portion, which has an important stabilizing function of the glenohumeral joint in priority exercises such as the bench press and dumbbell fly.

\section{Conclusion}

Finally, the use of SP exercise increased $\mathrm{AD}$ muscle activity when compared to the $\mathrm{BP}$ and DF. As for the MD, both the SP and LR exercises resulted in greater muscle activity, although the LR resulted in slightly superior muscle activity when compared to the SP. As for the PD, LR exercise presented higher muscle activity compared to the SP. Both the BP and DF resulted in low muscle recruitment in medial and posterior portions of the deltoid, possibly due to their stabilizing function in these exercises. In addition, further studies should include an analysis of the external kinematics structure to verify all the characteristics of the movements involved in the exercises evaluated (Król and Golas, 2017).

\section{Acknowledgements}

We would like to thank all the participants who selflessly participated in the study.

\section{References}

Andersen V, Fimland MS, Wiik E, Skoglund A, Saeterbakken AH. Effects of grip width on muscle strength and activation in the lat pull-down. J Strength Cond Res, 2014; 28: 1135-1142

Baechle TR, Earle RW. Essentials of strength training and conditioning. Champaign, IL: Human kinetics; 2008

Behm DG, Leonard AM, Young WB, Bonsey WA, MacKinnon SN. Trunk muscle electromyographic activity with unstable and unilateral exercises. J Strength Cond Res, 2005; 19: 193-201

Botton C, Wilhelm E, Ughini C, Pinto R, Lima C. Electromyographical analysis of the deltoid between different strength training exercises. Med Sport, 2013; 17: 67-71

Brzycki M. Strength testing - predicting a one-rep max from reps-to-fatigue. JOPERD, 1993; 64: 88-90

Castelein B, Cagnie B, Parlevliet T, Cools A. Superficial and deep scapulothoracic muscle electromyographic activity during elevation exercises in the scapular plane. J Orthop Sports Phys Ther, 2016; 46: 184-193

Ema R, Sakaguchi M, Akagi R, Kawakami Y. Unique activation of the quadriceps femoris during single-and multi-joint exercises. Eur J Appl Physiol, 2016; 116: 1031-1041

Franke AR, Botton C, Rodrigues R, Pinto R, Lima C. Analysis of anterior, middle and posterior deltoid activation during single and multijoint exercises. J Sports Med Phys Fitness, 2015; 55: 714-721 
Golas A, Maszczyk A, Pietraszewski P, Stastny P, Tufano J, Zajac A. Effects of pre-exhaustion on the patterns of muscular activity in the flat bench press. J Strength Cond Res, 2017; 31: 1919-1924

Gołaś A, Zwierzchowska A, Maszczyk A, Wilk M, Stastny P, Zając A. Neuromuscular control during the bench press movement in an elite disabled and able-bodied athlete. J Hum Kinet, 2017; 60: 209-215

Gołaś A, Maszczyk A, Pietraszewski P, Wilk M, Stastny P, Strońska K, Studencki M, Zając A. Muscular activity patterns of female and male athletes during the flat bench press. Biol Sport, 2018; 35: 175-179

Grgic J, Schoenfeld BJ, Davies TB, Lazinica B, Krieger JW, Pedisic Z. Effect of resistance training frequency on gains in muscular strength: a systematic review and meta-analysis. Sports Med, 2018; 48: 1-14

Hall SJ. Basic Biomechanics. New York, NY: McGraw-Hill Higher Education; 2006

Hermens HJ, Freriks B, Merletti R, Stegeman D, Blok J, Rau G, Disselhorst-Klug C, Hägg G. European recommendations for surface electromyography. $R R D, 1999 ; 8: 13-54$

Houglum PA, Bertoti DB. Brunnstrom's clinical kinesiology Philadelphia, PA: FA Davis Co; 2011

Junior V, Gentil P, Oliveira E, Carmo J. Comparison among the EMG activity of the pectoralis major, anterior deltoidis and triceps brachii during the bench press and peck deck exercises. Rev Bras Med Esporte, 2007; 13: 43-46

Król H, Gołaś A. Effect of barbell weight on the structure of the flat bench press. J Strength Cond Res, 2017; 31: 1321-1337

Liu J, Hughes R, Smutz W, Niebur G, Nan-An K. Roles of deltoid and rotator cuff muscles in shoulder elevation. Clin Biomech, 1997; 12: 32-38

McCaw ST, Friday JJ. A comparison of muscle activity between a free weight and machine bench press. $J$ Strength Cond Res, 1994; 8: 259-264

Muyor JM, Rodríguez-Ridao D, Martín-Fuentes I, Antequera-Vique JA. Evaluation and comparison of electromyographic activity in bench press with feet on the ground and active hip flexion. PloS One, 2019; 14: e0218209

NSCA Certification Commission. Exercise technique manual for resistance training. Champaign, IL: Human Kinetics; 2008

Rhea MR. Determining the magnitude of treatment effects in strength training research through the use of the effect size. J Strength Cond Res, 2004; 18: 918-920

Saeterbakken AH, Fimland MS. Effects of body position and loading modality on muscle activity and strength in shoulder presses. J Strength Cond Res, 2013; 27: 1824-1831

Schick EE, Coburn JW, Brown LE, Judelson DA, Khamoui AV, Tran TT, Uribe BP. A comparison of muscle activation between a Smith machine and free weight bench press. J Strength Cond Res, 2010; 24: 779784

Schoenfeld BJ, Peterson MD, Ogborn D, Contreras B, Sonmez G. Effects of low-vs. high-load resistance training on muscle strength and hypertrophy in well-trained men. J Strength Cond Res, 2015; 29: 29542963

Schoenfeld BJ, Pope ZK, Benik FM, Hester GM, Sellers J, Nooner JL, Schnaiter JA, Bond-Williams KE, Carter AS, Ross CL, Just BL, Henselmans M, Krieger JW. Longer interset rest periods enhance muscle strength and hypertrophy in resistance-trained men. J Strength Cond Res, 2016; 30: 1805-1812

Schwanbeck S, Chilibeck PD, Binsted G. A comparison of free weight squat to smith machine squat using electromyography. J Strength Cond Res, 2009; 23: 2588-2591

Stastny P, Gołaś A, Blazek D, Maszczyk A, Wilk M, Pietraszewski P, Petr M, Uhlir P, Zając A. A systematic review of surface electromyography analyses of the bench press movement task. PloS One, 2017; 12: e0171632

Van den Tillaar R, Ettema G. A comparison of successful and unsuccessful attempts in maximal bench pressing. Med Sci Sports Exerc, 2009; 41: 2056

Vilaça-Alves J, Geraldes L, Fernandes HM, Vaz L, Farjalla R, Saavedra F, Reis VM. Effects of pre-exhausting the biceps brachii muscle on the performance of the front lat pull-down exercise using different handgrip positions. J Hum Kinet, 2014; 42: 157-163 
Vigotsky AD, Halperin I, Lehman GJ, Trajano GS, Vieira TM. Interpreting signal amplitudes in surface electromyography studies in sport and rehabilitation sciences. Front Physiol, 2018; 8: 985

Welsch EA, Bird M, Mayhew JL. Electromyographic activity of the pectoralis major and anterior deltoid muscles during three upper-body lifts. J Strength Cond Res, 2005; 19: 449

Wickham J, Pizzari T, Stansfeld K, Burnside A, Watson L. Quantifying 'normal'shoulder muscle activity during abduction. J Electromyogr Kinesiol, 2010; 20(2): 212-222

Wilk M, Golas A, Stastny P, Nawrocka M, Krzysztofik M, Zajac A. Does tempo of resistance exercise impact training volume? J Hum Kinet, 2018; 62: 241-250

Wilk M, Gepfert M, Krzysztofik M, Golas A, Mostowik A, Maszczyk A, Zajac A. The Influence of Grip Width on Training Volume During the Bench Press with Different Movement Tempos. J Hum Kinet, 2019; 68: 49-57

Wright GA, Delong TH, Gehlsen G. Electromyographic activity of the hamstrings during performance of the leg curl, stiff-leg deadlift, and back squat movements. J Strength Cond Res, 1999; 13: 168-174

\section{Corresponding author:}

\section{Yuri de Almeida Costa Campos.}

Department of Physical Education, University of Lavras. Zip Code: 37200-000, PO BOX 3037, Lavras, Brazil. Phone Number: +55(35) 3829-5132.

Email: reiclauy@hotmail.com 